\title{
ON SYMMETRIC BERNOULLI CONVOLUTIONS
}

\section{BY AUREL WINTNER}

In a previous note* a class of sequences $\left\{a_{n}\right\}$ has been determined such that

$$
\begin{aligned}
\prod_{n=1}^{\infty} \cos \left(a_{n} t\right) & =O\left(\exp \left(-A t^{\gamma}\right)\right) \text { as } t \rightarrow+\infty, \\
a_{n} & >0, \sum_{n=1}^{\infty} a_{n}^{2}<+\infty,
\end{aligned}
$$

where $A>0$ and $\gamma>0$. The role of this estimate was that of assuring a high degree of smoothness for the distribution function having the infinite product as Fourier-Stieltjes transform. In fact, this distribution function is an entire function if $\gamma>1$, it is regular analytic if $\gamma=1$ and it has derivatives of arbitrarily high order whenever there exists a $\gamma(>0)$. For the sequences $\left\{a_{n}\right\}$ in question and also for some more general sequences a simple proof of (1) will be given by means of an appraisal indicated in the reference cited. This appraisal has the advantage of being valid for every $\left\{a_{n}\right\}$ and it gives results also if (1) is not satisfied. It implies for instance the fact that the infinite product $\rightarrow 0$ as $t \rightarrow+\infty$ in cases of the type $a_{n}=c^{-n^{\delta}}$, where $c>1$ and $0<\delta<1$.

The $n$th factor of (1) does not come near to \pm 1 if $1 \leqq a_{n} t \leqq 2$, since this limitation implies $\left|\cos \left(a_{n} t\right)\right|<2 / 3$. Thus

$$
\left|\prod_{n=1}^{\infty} \cos \left(a_{n} t\right)\right|<(2 / 3)^{K(t)},
$$

if $1 \leqq a_{n} t \leqq 2$ is satisfied for $K=K(t)$ values of $n$. Now

$$
K(t) \geqq N(2 t)-N(t),
$$

if $a_{n} \geqq 1 / t$ holds for exactly $N(t)$ values of $n$.

Thus if $a_{n}=n^{-\alpha}$, then $N(t)=\left[t^{1 / \alpha}\right]$, hence $K(t)>C t^{1 / \alpha}$ for

* A. Wintner, On analytic convolutions of Bernoulli distributions, American Journal of Mathematics, vol. 56 (1934), pp. 659-663. 
some $C>0$ so that (1) is satisfied by $\gamma=1 / \alpha$. Similarly, if $N(t) \sim t^{\beta} S(t)^{ \pm 1}$, where $\beta>0$ and $S(t)$ is, as in the reference cited, a "slow" function, then (1) holds for every $\gamma<\beta$. If $1 / a_{n}$ is the $n$th prime number, then (1) holds for every $\gamma<1$ since $K(t)$ $>C t^{\gamma}$ in virtue of the elementary inequalities of Tchebycheff. Thus it is not necessary to use the prime number theorem $\beta=1$, $S(t)^{ \pm 1}=1 / \log t$, applied in the reference cited. Correspondingly, the present method enables us to prove (1) also for sequences for which $N(t)$ is not $\sim t^{\beta} S(t)^{ \pm 1}$.

The Johns Hopkins University

\section{PROOF OF THREE PROPOSITIONS OF PALEY}

BY M. FEKETE

1. Introduction. In a letter to Fejér,* Paley stated three interesting propositions concerning Fourier series of bounded and continuous functions whose Fourier coefficients satisfy the conditions $n a_{n}, n b_{n} \geqq-K, K \geqq 0$. The letter of Paley contains only a very brief sketch of the proof. After knowing Paley's results the author succeeded in developing complete proofs with various improvements of the estimates, and even in extending them to a wider class of Fourier series of almost periodic functions. These extensions will be treated elsewhere. In the present note we prove the following three theorems.

It will be assumed throughout that $f(x)$ is real-valued and periodic, of period $2 \pi$, and Lebesgue integrable over $(-\pi, \pi)$. Let

$$
f(x) \sim a_{0}+\sum_{n=1}^{\infty}\left(a_{n} \cos n x+b_{n} \sin n x\right)
$$

be its Fourier series expansion and let

$$
s_{n}=s_{n}(x)=a_{0}+\sum_{\nu=1}^{n}\left(a_{\nu} \cos \nu x+b_{\nu} \sin \nu x\right)
$$

* This letter is reproduced in a note by Fejér, On a theorem of Paley, this Bulletin, vol. 40 (1934), pp. 469-475, especially pp. 474-475. It was communicated to the author by Professor Fejér in September, 1933. 